\title{
Stratégies de conception pour optimiser la transmission Slide-o-Cam
}

\author{
Damien Chablat ${ }^{1, a}$ et Jorge Angeles ${ }^{2}$ \\ 1 Institut de recherche en communications et cybernétique de Nantes*, UMR CNRS 6597, 1 rue de la Noë, \\ 44321 Nantes, France \\ ${ }^{2}$ Department of mechanical engineering \& centre for intelligent machines, McGill University, 817 Sherbrooke Street West, \\ Montreal, Canada H3A 2K6
}

Reçu le 8 septembre 2005, accepté le 26 mai 2006

\begin{abstract}
Résumé - L'optimisation de l'angle de pression dans la transmission Slide-o-Cam est rapportée dans cet article. Cette transmission est basée sur le mécanisme Slide-o-Cam, un mécanisme à cames associées à des galets montés sur une glissière. Dans cette transmission, le mouvement est transmis via un contact de pur roulement sans glissement. Ainsi, les frottements sont moins importants que dans les transmissions à crémaillère ou à vis à billes. L'angle de pression est un indice de performance approprié pour caractériser cette transmission parce qu'il caractérise la force transmise entre une came et un galet. Deux stratégies de conception sont étudiées, à savoir, (i) l'augmentation du nombre de lobes sur chaque came et (ii) l'augmentation du nombre de cames. Cette transmission est conçue pour remplacer les vis à billes actuellement utilisées par l'orthoglide, un robot parallèle à trois degrés de liberté développé à l'École Centrale de Nantes pour des applications d'usinage à grande vitesse.
\end{abstract}

Mots clés : Conception optimale / Slide-o-Cam / angle de pression / orthoglide

Abstract - Strategies for the design optimisation of the Slide-o-Cam transmission. The optimization of the pressure angle in a cam-follower transmission is reported in this paper. This transmission is based on Slide-o-Cam, a cam mechanism with multiple rollers mounted on a common translating follower. The design of Slide-o-Cam, a transmission intended to produce a sliding motion from a turning drive, or vice versa, was reported elsewhere. This transmission provides pure-rolling motion, thereby reducing the friction of rack-and-pinions and linear drives. The pressure angle is a suitable performance index for this transmission because it determines the amount of force transmitted to the load vs. that transmitted to the machine frame. Two alternative design strategies are studied, namely, $(i)$ increase the number of lobes on each cam or $(i i)$ increase the number of cams. This device is intended to replace the current ball-screws in orthoglide, a three-DOF parallel robot for the production of translational motions, currently under development at École Centrale de Nantes for machining applications.

Key words: Design optimization / Slide-o-Cam / pressure angle / orthoglide

\section{Introduction}

Généralement, la conversion d'un mouvement de rotation en un mouvement de translation est réalisée au moyen d'une vis à billes ou d'une crémaillère. La première solution est très répandue mais a cependant quelques faiblesses. En effet, elle comprend un nombre élevé de pièces

\footnotetext{
a Auteur correspondant :

Damien.Chablat@irccyn.ec-nantes.fr

* IRCCyN : UMR 6597 CNRS, École Centrale de Nantes, Université de Nantes, École des Mines de Nantes, 44321 Nantes, France
}

mobiles puisqu'elle comprend plusieurs billes circulant dans un chemin de roulement usiné sur un axe. En outre, la portance générée par cette solution est faible du fait de la forme ponctuelle du contact entre les billes et l'axe de la transmission. En ce sens, les actionneurs linéaires sont plus intéressants puisque la transmission se fait dans ce cas sans contact au niveau du moteur et ils peuvent être associés à un guidage par roulements. Cependant, cette transmission est onéreuse et demande beaucoup d'entretien puisqu'elle présente une succession de bi-pôles. De plus, elles génèrent deux types de pollution, thermique et magnétique. Dans le premier cas, cela nuit à la précision 


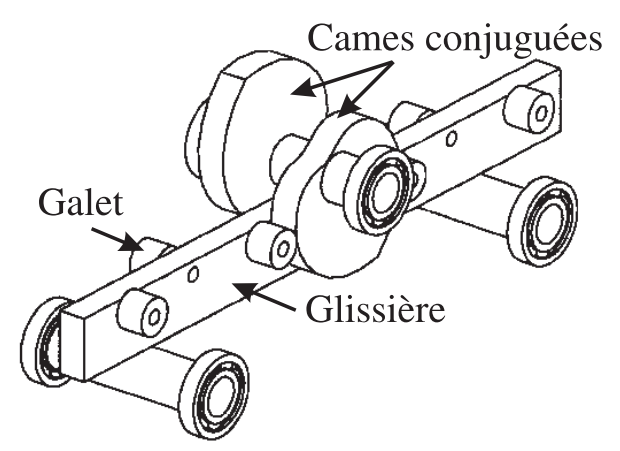

Fig. 1. Schéma de Slide-o-Cam.

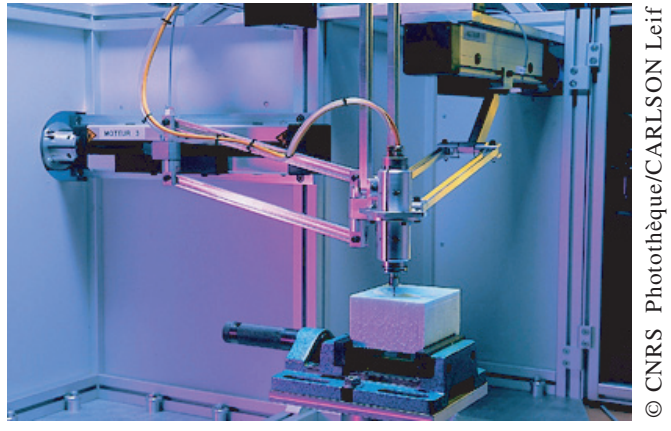

Fig. 2. L'orthoglide. dimensionnelle de la machine et dans le second cas, cela pose des problèmes lorsque l'on usine des matériaux ferreux.

Une nouvelle transmission, appelée Slide-o-Cam, a été présentée dans [1] (Fig. 1) pour transformer un mouvement de rotation en mouvement de translation. Slide-oCam se compose de quatre éléments principaux : $(i)$ le bâti, (ii) les cames, (iii) la glissière et (iv) les galets. L'axe d'entrée sur lequel la came est montée est appelé arbre à cames et tourne à une vitesse angulaire variable grâce à un moteur rotatif commandé par ordinateur. La puissance est transmise à la glissière au moyen du contact entre les cames et les galets. Ce contact a la particularité d'être de type « pur roulement sans glissement ». En outre, le galet est composé de deux éléments : un axe et un roulement. Le roulement est monté à l'une des extrémités de l'axe, alors que l'autre extrémité est encastrée dans la glissière. Un contact linéique existe entre la came et le galet sur la surface externe du roulement. Par ailleurs, Slideo-Cam est constitué de deux cames conjuguées et de deux séries de galets assurant alternativement la transmission du mouvement; les galets sont ainsi entraînés par deux cames successivement pour réaliser un cycle complet. Les avantages principaux de l'utilisation d'un mécanisme de type came-galet pour la transformation d'un mouvement de rotation en un mouvement de translation pure est la réduction de frottements, des efforts de contact, de jeu (backlash), et ainsi d'usure.

Après optimisation, cette transmission pourra remplacer les trois vis à billes actuellement utilisées par le prototype de l'orthoglide [2]. L'orthoglide comporte trois liaisons prismatiques montées orthogonalement, trois jambes identiques et une plate-forme mobile, qui se déplace dans l'espace cartésien avec l'orientation fixe (Fig. 2). Les trois moteurs sont des moteurs SANYO DENKI (réf. P30B08075D) avec un couple constant de 1,2 Nm de 0 à 3000 tr.min ${ }^{-1}$. Cette propriété permet au mécanisme de déplacer une charge de $4 \mathrm{~kg}$ et de fournir une accélération minimale de $17 \mathrm{~m} . \mathrm{s}^{-2}$ et une vitesse minimale de $1,3 \mathrm{~m} . \mathrm{s}^{-1}$ dans tout son espace de travail. En outre, l'avance est de $50 \mathrm{~mm}$ par tour de came, alors que le rayon minimum de l'arbre à cames est de $8,5 \mathrm{~mm}$.

À la différence de Lampinen [3], qui a utilisé un algorithme génétique, ou Zhang et Shin [4], qui ont employé ce qu'ils ont appelé « la méthode du mouvement relatif », où la vitesse et l'accélération relatives du galet et de la came sont prescrites, nous employons une méthode déterministe qui tient compte des contraintes géométriques et d'usinage [5].

L'angle de pression $\mu$ (ou son complément, l'angle de transmission) est un indice de performance pouvant caractériser les transmissions came/galet. Une définition de l'angle de pression est l'angle aigu défini par la normale commune au point de contact entre la came et le galet et la direction de déplacement de la glissière [6].

À la différence de Carra et al. [7], qui ont utilisé un rayon de courbure négatif pour réduire l'angle de pression, nous utilisons un rayon de courbure positif qui nous permet d'assembler plusieurs galets sur la même glissière. Pour optimiser l'angle de pression, nous allons étudier deux différentes stratégies de conception, à savoir, $(i)$ augmenter le nombre de lobes sur chaque came ou (ii) augmenter le nombre de cames. Les relations définies dans [8] pour des profils de came avec un seul lobe sont généralisées pour les cames à plusieurs lobes.

\section{Synthèse du mécanisme à came planaire}

Soit $x-y$ un repère fixe lié à la machine et $u-v$ un repère lié à la came (Fig. 3). $O_{1}$ est l'origine de ces deux repères, alors que $\mathrm{O}_{2}$ est le centre de chaque galet et $C$ est le point de contact entre la came et le galet. Les paramètres géométriques définissant le mécanisme à cames, avec $n$ lobes, sont illustrés dans la même figure. La notation de cette figure est basée sur la notation présentée dans [9] et complétée dans [8], à savoir,

- $p$, le pas de la transmission, c'est-à-dire la distance entre le centre de deux galets alignés sur une même came;

- $n$, le nombre de lobes de chaque came;

- e, la distance entre l'axe de la came et la ligne des centres des galets;

- $a$, le rayon du galet;

- $\psi$, l'angle de rotation de la came, c'est-à-dire l'entrée du mécanisme;

- $s$, le déplacement de l'axe de la came, donné par la position de $O_{1}$, c'est-à-dire la sortie du mécanisme;

- $\mu$, l'angle de pression; 


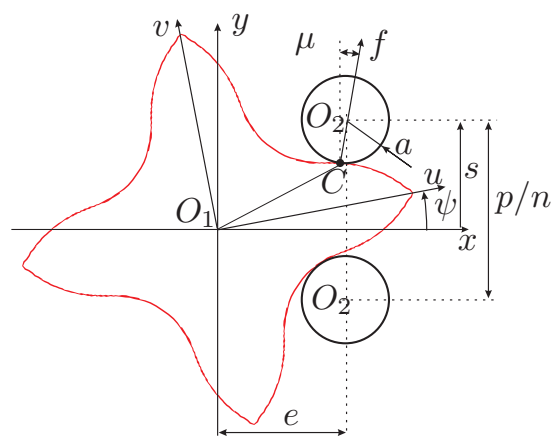

Fig. 3. Les paramètres de Slide-O-Cam.

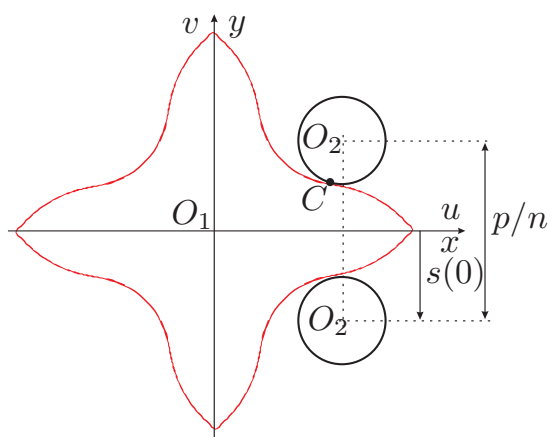

Fig. 4. Configuration initiale, pour une came avec quatre lobes.

- $f$, la force transmise de la came sur le galet.

Dans notre conception, $p$ est placé à $50 \mathrm{~mm}$, afin de répondre aux caractéristiques de l'orthoglide.

\subsection{Fonction de transfert de Slide-o-Cam}

Les paramètres ci-dessus comme la surface de contact sur la came sont déterminés par les relations géométriques dictées par le théorème d'Aronhold-Kennedy dans le plan [10]. Quand la came fait un tour complet $(\Delta \psi=2 \pi)$, le déplacement des galets est égal à $p$, c'est-à-dire la distance entre $n+1$ galets $(\Delta s=p)$. En outre, si nous considérons la configuration initiale des galets, comme représentée dans la figure 4 , un galet est au-dessous de l'axe $x$ pour $\psi=0$, de sorte que nous ayons $s(0)=$ $-p /(2 n)$. Par conséquent, l'expression pour la fonction de transfert de Slide-o-Cam $s$ est

$$
s(\psi)=\frac{p}{2 \pi} \psi-\frac{p}{2 n}
$$

Les expressions de la première et de la seconde dérivée de $s(\psi)$ par rapport à $\psi$ peuvent être facilement calculées,

$$
s^{\prime}(\psi)=\frac{p}{2 \pi} \quad \text { et } \quad s^{\prime \prime}(\psi)=0
$$

\subsection{Détermination du profil de la came}

Le profil de la came est déterminé par le déplacement du point de contact $C$ autour de la came. Ce contact entre un lobe et un galet a lieu dans l'intervalle $0 \leq \psi \leq 2 \pi / n$. Pour cet intervalle, nous trouvons le profil d'un lobe. Les lobes restants sont trouvés par rotation autour de $O_{1}$. Les coordonnées cartésiennes de $C$ dans le repère $u-v$ sont les suivantes [9]

$$
\begin{aligned}
& u_{\mathrm{c}}(\psi)=b_{1} \cos (\psi)+\left(b_{2}-a\right) \cos (\delta-\psi) \\
& v_{\mathrm{c}}(\psi)=-b_{1} \sin (\psi)+\left(b_{2}-a\right) \sin (\delta-\psi)
\end{aligned}
$$

avec des coefficients $b_{1}, b_{2}$ et $\delta$ donnés par

$$
\begin{aligned}
b_{1} & =-s^{\prime}(\psi) \sin \alpha \\
b_{2} & =\sqrt{\left[e+s^{\prime}(\psi) \sin \alpha\right]^{2}+[s(\psi) \sin \alpha]^{2}} \\
\delta & =\arctan \left(\frac{-s(\psi) \sin \alpha}{e+s^{\prime}(\psi) \sin \alpha}\right)
\end{aligned}
$$

avec $\alpha$ l'angle formé par l'axe de la came et la direction de translation des galets; $\alpha$ est positif dans le sens trigonométrique. À partir de la convention de signe adoptée pour l'angle d'entrée $\psi$ et la sortie $s$, comme représenté dans la figure 3 , nous avons

$$
\alpha=-\pi / 2
$$

Nous présentons maintenant un paramètre de conception sans dimension $\eta$, qui sera maintenant utilisé :

$$
\eta=\frac{e}{p}
$$

Ainsi, à partir des équations (1), (2a), (3a)-(3c), (4) et (5), nous écrivons les expressions des coefficients $b_{1}, b_{2}$ et $\Delta$

$$
\begin{aligned}
b_{1} & =\frac{p}{2 \pi} \\
b_{2} & =\frac{p}{2 \pi} \sqrt{(2 \pi \eta-1)^{2}+\left(\psi-\frac{\pi}{n}\right)^{2}} \\
\Delta & =\arctan \left(\frac{n \psi-\pi}{2 n \pi \eta-n}\right)
\end{aligned}
$$

d'où une première contrainte sur $\eta$ est dérivée, $\eta \neq$ $1 /(2 \pi)$. Avec cette définition cependant, le profil de la came n'est pas fermé.

Un angle étendu nommé $\Delta$ est présenté par [11], pour que le profil de la came soit fermé. L'angle $\Delta$ est l'une des racines de l'équation $v_{\mathrm{c}}$. Dans le cas de Slide-o-Cam, $\Delta$ est négatif, comme montré dans la figure 5 pour une came avec un lobe. Le contact entre le profil de la came et un galet est créé (respectivement perdu) pour $\psi=\Delta$ ou $\psi=2 \pi-\Delta$ comme représenté dans les figures 5 (b et $\mathrm{c}$ ).

En conséquence, le profil de la came est fermé lorsque $\Delta \leq \psi \leq 2 \pi / n-\Delta$, avec une relation équivalente pour les $n-1$ lobes restants.

\subsection{Détermination du profil caractéristique}

Le profil caractéristique est défini par la trajectoire du centre $\mathrm{O}_{2}$ des galets. Celle-ci est différente de la trajectoire du point de contact $C$ qui donne le profil de la came. 


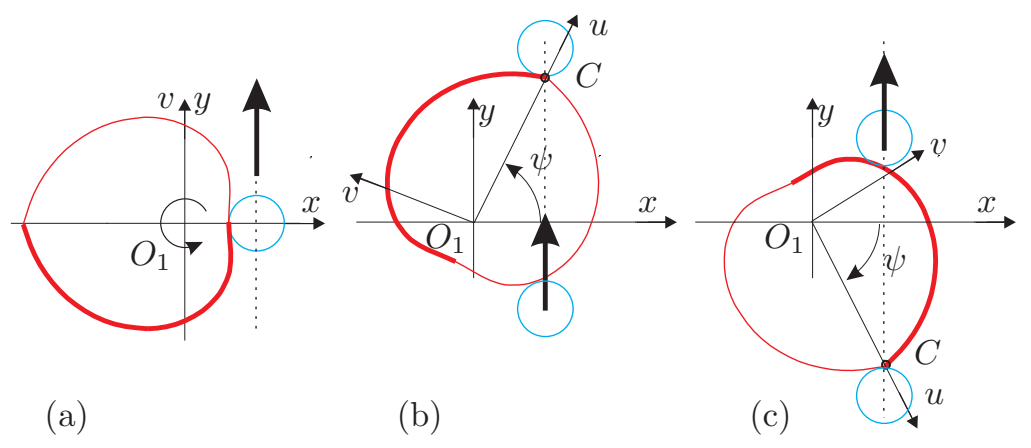

Fig. 5. Les trois racines de $v_{\mathrm{c}}$ en fonction $\psi$, pour une came avec un seul lobe : (a) $\psi=\pi$; (b) $\psi=2 \pi-\Delta$; et (c) $\psi=\Delta$.

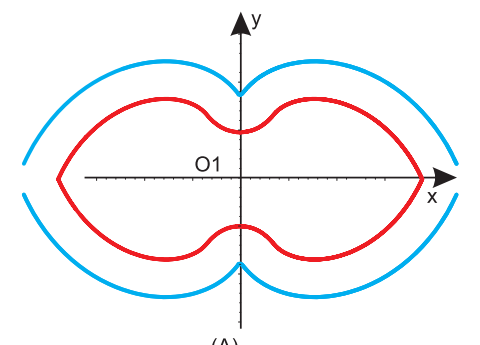

(A)

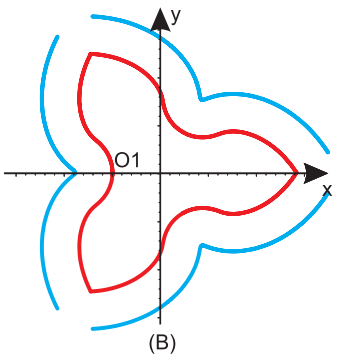

(B)

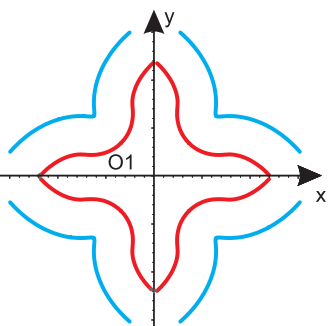

(C)

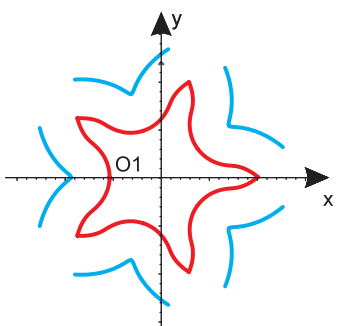

(D)

Fig. 6. Profil de la came (en rouge) et le profil caractéristique (en bleu), pour $\Delta \leq \psi \leq 2 \pi / n-\Delta$, avec $a=4$, $p=50$ et $e=9$, for : (a) $n=2$; (b) $n=3$; (c) $n=4$; et (d) $n=5$.

Les coordonnées cartésiennes du point $\mathrm{O}_{2}$ dans le repère $x-y$ sont $(e, s)$ (Fig. 3). Par conséquent, les coordonnées cartésiennes du profil caractéristique dans le repère mobile $u-v$ sont

$$
\begin{aligned}
& u_{\mathrm{p}}(\psi)=e \cos (\psi)+s(\psi) \sin (\psi) \\
& v_{\mathrm{p}}(\psi)=-e \sin (\psi)+s(\psi) \cos (\psi)
\end{aligned}
$$

La figure 6 représente le profil de la came et le profil caractéristique associé lorsque $\Delta \leq \psi \leq 2 \pi / n-\Delta$ pour $n$ lobes.

\subsection{Contraintes géométriques sur le mécanisme}

Pour obtenir un mécanisme réalisable, le rayon du galet $a$ doit satisfaire deux conditions, comme montré dans la figure 7 :

- Deux galets consécutifs du même côté de la transmission ne doivent pas interférer l'un dans l'autre. Comme $p / n$ est la distance entre le centre de deux galets consécutifs, nous avons comme contrainte $2 a<p / n$. Par conséquent la première condition sur $a$ est

$$
a<\frac{p}{2 n}
$$

- Le rayon $b$ de l'axe moteur sur lequel les cames sont montées doit aussi être pris en compte. Par conséquent, nous avons la contrainte $a+b \leq e$,
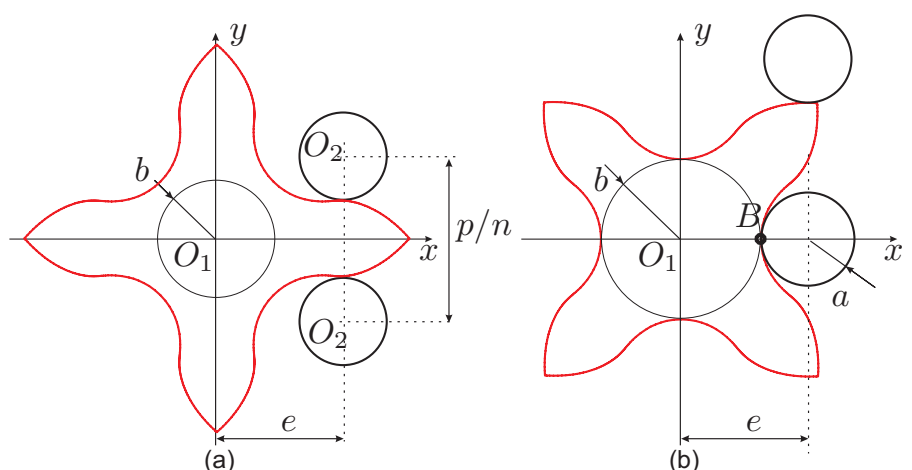

Fig. 7. Contraintes sur le rayon du galet : (a) $a<p /(2 n)$; et (b) $a \leq \eta p-b$.

la deuxième contrainte pour $a$ en termes de paramètre $\eta$ est $^{1}$

$$
a \leq \eta p-b
$$

Vu la configuration initiale du galet représentée dans la figure 4 , la composante $v$ de la coordonnée cartésienne du point de contact $C$ est négative, c'est-à-dire $v_{\mathrm{c}}(0) \leq$ 0 . Cependant, à partir de l'expression de $v_{\mathrm{c}}(\psi)$ pour les paramètres $b_{2}$ et $\Delta$ donnés dans les équations (6b) et (6c),

\footnotetext{
${ }^{1}$ Il est possible d'avoir $a=\eta p-b$ si les cames et l'axe sont usinés dans un même bloc. Ainsi, il y a un point commun (Fig. 7b), entre l'axe moteur et le profil de la came. Ce ne sera pas la solution choisie pour notre conception, parce qu'il est trop compliqué pour usiner les cames et l'axe moteur en même temps.
} 
respectivement, la relation ci-dessus mène à la condition suivante

$$
\begin{aligned}
&\left(\frac{p}{2 \pi n} \sqrt{(2 n \pi \eta-n)^{2}+\pi^{2}}-a\right) \\
& \times \sin \left[\arctan \left(\frac{-\pi}{2 n \pi \eta-n}\right)\right] \leq 0
\end{aligned}
$$

À partir de l'expression précédente, nous définissons les paramètres $A$ et $B$

$$
A=\frac{p}{2 n \pi} \sqrt{(2 n \pi \eta-n)^{2}+\pi^{2}}-a
$$

et

$$
B=\sin \left[\arctan \left(\frac{-\pi}{2 n \pi \eta-n}\right)\right]
$$

Maintenant nous dérivons la contrainte $A$ et puisque $(2 \pi n \eta-n)^{2}>0$, nous avons

$$
\sqrt{(2 n \pi \eta-n)^{2}+\pi^{2}}>\pi
$$

Par conséquent,

$$
A>\frac{p}{2 n}-a
$$

En outre, à partir de la contrainte $a$ définie dans l'équation (8), nous avons $p /(2 n)-a>0$, d'où $A>0$. En conséquence, la contrainte $v_{\mathrm{c}}(0) \leq 0$ mène à la contrainte $B \leq 0$.

Nous transformons maintenant l'expression de $B$ en utilisant la relation trigonométrique suivante

$$
\forall x \in \mathbb{R}, \sin (\arctan x)=\frac{x}{\sqrt{1+x^{2}}}
$$

Par conséquent, la contrainte $v_{\mathrm{c}}(0) \leq 0$ devient

$$
\frac{-\pi}{n(2 \pi \eta-1) \sqrt{1+\pi^{2} /(2 n \pi \eta-n)^{2}}} \leq 0
$$

ce qui est vrai seulement si $2 \pi \eta-1>0$. En conclusion, la contrainte $v_{\mathrm{c}}(0) \leq 0$ mène à une contrainte sur $\eta^{2}$,

$$
\eta>\frac{1}{2 \pi}
$$

Les équations (8), (9) et (10) nous permettent de réduire l'espace des paramètres de conception. La figure 8 représente l'espace des paramètres de conception pour $p=50 \mathrm{~mm}$ et $b=4,25 \mathrm{~mm}$. Nous pouvons noter que lorsque le nombre de lobes de la came augmente, la valeur maximale de a diminue.

\footnotetext{
${ }^{2} \mathrm{Si}$ dans la configuration initiale le galet était du côté supérieur du l'axe $x$, la fonction d'entrée-sortie serait $s(\psi)=$ $(p / 2 \pi) \psi+p / 2$, et nous aurions la contrainte $v_{\mathrm{c}}(0) \geq 0$, qui mènerait à la même contrainte $\eta>1 /(2 \pi)$.
}

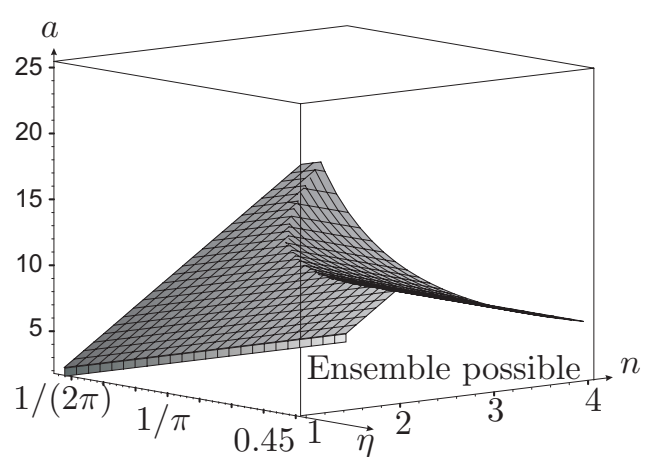

Fig. 8. Paramètres de conception pour $p=50 \mathrm{~mm}$ et $b=$ $4,25 \mathrm{~mm}$.

\subsection{L'angle de pression}

L'angle de pression des transmissions à cames est défini par la normale commune au point de contact $C$ entre la came et le galet et la direction de déplacement du point $C$ par rapport de la glissière [12], comme représenté dans la figure 3 , où l'angle de pression est noté $\mu$. Cet angle joue un rôle important dans la conception des transmissions à cames. Plus $|\mu|$ est petit, meilleure est la qualité de la transmission. Dans le cas d'opérations à grande vitesse, c'est-à-dire pour des vitesses angulaires des cames supérieures à 50 tr.min ${ }^{-1}$, l'angle de pression doit être inférieur à $30^{\circ}$.

L'expression de l'angle de pression $\mu$ est donnée dans $[12]$

$$
\tan \mu=\frac{s^{\prime}(\psi)-e}{s(\psi)}
$$

À partir des expressions de $s$ et $s^{\prime}$, et en utilisant le paramètre $\eta$ donné dans l'équation (1), (2a) et (6), respectivement, l'expression pour l'angle de pression devient

$$
\tan \mu=\frac{n-2 n \pi \eta}{n \psi-\pi}
$$

\subsection{Cames conjuguées}

Pour réduire l'angle de pression, plusieurs cames peuvent être assemblées sur le même arbre. Nous notons $m$ le nombre de cames et $\beta$ l'angle de la rotation entre deux cames adjacentes, c'est-à-dire,

$$
\beta=\frac{2 \pi}{n m}
$$

Sur le mécanisme Slide-o-Cam initialement conçu dans [1], deux cames conjuguées avec un lobe chacune et $\beta=\pi$ ont été utilisées. La figure 9 montre deux profils de came avec un et deux lobes chacune.

\subsection{Convexité du profil de la came}

La convexité de la came influence la précision de l'usinage. Ainsi, il est préférable d'avoir un profil de la came 


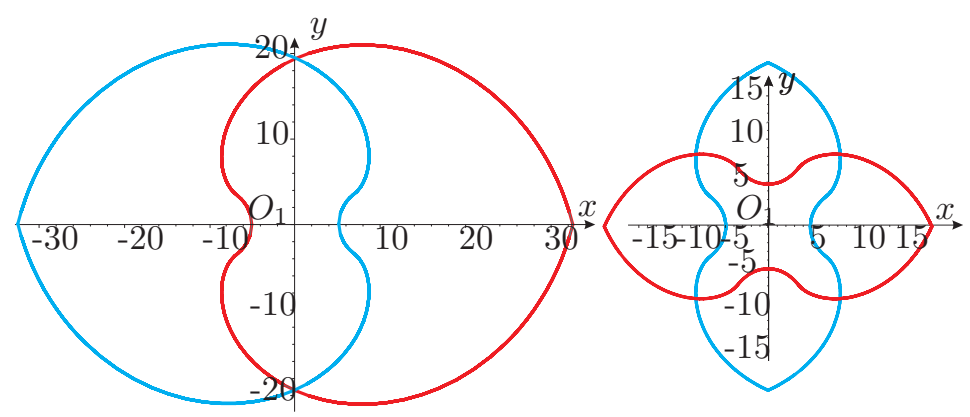

(a)

(b)

Fig. 9. Assemblage de deux cames avec $p=50 \mathrm{~mm}, a=10 \mathrm{~mm}$ et $e=9 \mathrm{~mm}$ : (a) un lobe; (b) deux lobes.

convexe pour chaque lobe lorsqu'elle mène un galet. Dans cette section, nous établissons les conditions sur les paramètres de conception $\eta$ et $a$ afin d'obtenir une telle propriété. Nous étudierons ainsi le signe de la courbure du profil de la came par l'intermédiaire du profil caractéristique.

La courbure de n'importe quelle courbe paramétrique planaire, dans le repère cartésien $u-v$ et en fonction de $\psi$, est donnée dans [12],

$$
\kappa=\frac{v^{\prime}(\psi) u^{\prime \prime}(\psi)-u^{\prime}(\psi) v^{\prime \prime}(\psi)}{\left[u^{\prime}(\psi)^{2}+v^{\prime}(\psi)^{2}\right]^{3 / 2}}
$$

Le signe de $\kappa$ dans l'équation (11) indique si la courbe est convexe ou concave en un point : un signe positif de $\kappa$ implique une convexité, alors qu'un signe négatif de $\kappa$ implique une concavité. Le calcul de la première et de la seconde dérivée des coordonnées cartésiennes du profil caractéristique, défini dans l'équation (7b), par rapport à l'angle de la rotation de la came $\psi$, sont

$$
\begin{aligned}
u_{\mathrm{p}}^{\prime}(\psi) & =\left(s^{\prime}(\psi)-e\right) \sin (\psi)+s(\psi) \cos (\psi) \\
v_{\mathrm{p}}^{\prime}(\psi) & =\left(s^{\prime}(\psi)-e\right) \cos (\psi)-s(\psi) \sin (\psi) \\
u_{\mathrm{p}}^{\prime \prime}(\psi) & =\left(2 s^{\prime}(\psi)-e\right) \cos (\psi)-s(\psi) \sin (\psi) \\
v_{\mathrm{p}}^{\prime \prime}(\psi) & =-\left(2 s^{\prime}(\psi)-e\right) \sin (\psi)-s(\psi) \cos (\psi)
\end{aligned}
$$

Par la substitution $\eta=e / p$, avec l'équation (12a)(12d) dans l'équation (11), la courbure $\kappa_{\mathrm{p}}$ du profil caractéristique est obtenue

$$
\kappa_{\mathrm{p}}=-\frac{2 n^{2} \pi}{p} \frac{\left[(n \psi-\pi)^{2}+2 n^{2}(2 \pi \eta-1)(\pi \eta-1)\right]}{\left[(n \psi-\pi)^{2}+n^{2}(2 \pi \eta-1)^{2}\right]^{3 / 2}}
$$

à condition que le dénominateur ne s'annule jamais pour n'importe quelle valeur de $\psi$, c'est-à-dire lorsque nous avons la condition :

$$
\eta \neq 1 /(2 \pi)
$$

À partir de l'expression de $\kappa_{\mathrm{p}}$ définie dans l'équation (13), nous prenons pour chaque valeur de $\psi$,

$$
\kappa_{\mathrm{p}} \geq 0 \quad \text { si } \quad(2 \pi \eta-1)(\pi \eta-1) \geq 0 \text { and } \eta \neq \frac{1}{2 \pi}
$$

d'où la condition pour $\eta$ :

$$
\kappa_{\mathrm{p}} \geq 0 \quad \text { si } \quad \eta \in\left[0, \frac{1}{2 \pi}\left[\cup \left[\frac{1}{\pi},+\infty[\right.\right.\right.
$$

La condition sur $\eta$ donnée dans l'équation (14) doit être combinée avec la condition établie dans l'équation (10), c'est-à-dire $\eta>1 /(2 \pi)$. Par conséquent, la condition finale sur la convexité du profil caractéristique est :

$$
\eta \geq \frac{1}{\pi}
$$

\section{Influence du nombre de cames conjuguées et du nombre de lobes sur l'angle de pression}

\subsection{Intervalle angulaire actif de l'angle de pression}

Dans notre étude, nous nous intéressons uniquement à l'intervalle sur $\psi$ où la came conduit un galet pour le déplacer vers la droite comme indiqué dans les figures 10 , 11 et 12 ; nous appelons ceci l'intervalle angulaire actif. Un autre intervalle de $\psi$ existe, avec la même amplitude, quand la came conduit un galet pour le déplacer vers la gauche comme est représenté dans les figures 10, 11 et 12 .

- Transmission à une came : L'intervalle angulaire actif est :

$$
\frac{\pi}{n} \leq \psi \leq \frac{2 \pi}{n}-\Delta
$$

En effet, si nous commençons le mouvement dans la configuration initiale représentée dans la figure 4, avec la came tournant dans le sens trigonométrique, la came commence à mener le galet seulement lorsque $\psi=\pi / n$; la came peut mener le galet jusqu'à ce que le contact soit perdu, c'est-à-dire lorsque $\psi=2 \pi / n-\Delta$, comme illustré dans la figure $5 \mathrm{c}$. Le mécanisme Slide-o-Cam avec une seule came ne peut pas mener le galet pendant un tour complet, comme représenté dans la figure 10. Ce résultat reste vrai pour toutes les valeurs de $n$. Ainsi, Slide-o-Cam doit posséder au moins deux cames conjuguées pour fonctionner. En conclusion, il existe un contact entre une came et un galet pouvant déplacer la glissière vers la droite uniquement dans l'intervalle $-\pi / n+\Delta \leq \psi \leq 2 \pi / n-\Delta$. 


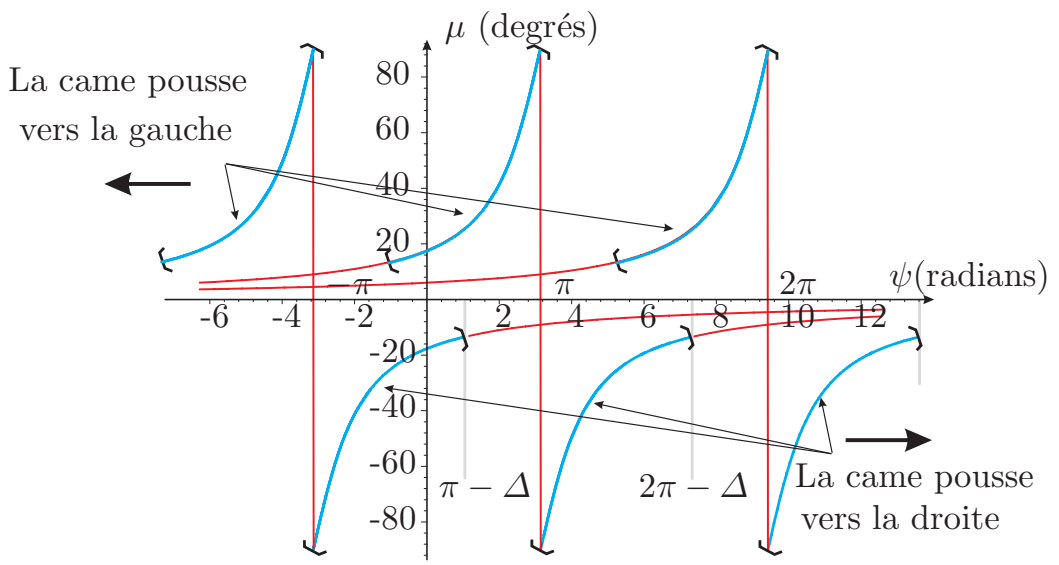

Fig. 10. Distribution de l'angle de pression pour une came avec un lobe, avec $p=50 \mathrm{~mm}, a=10 \mathrm{~mm}$ et $e=9 \mathrm{~mm}$.

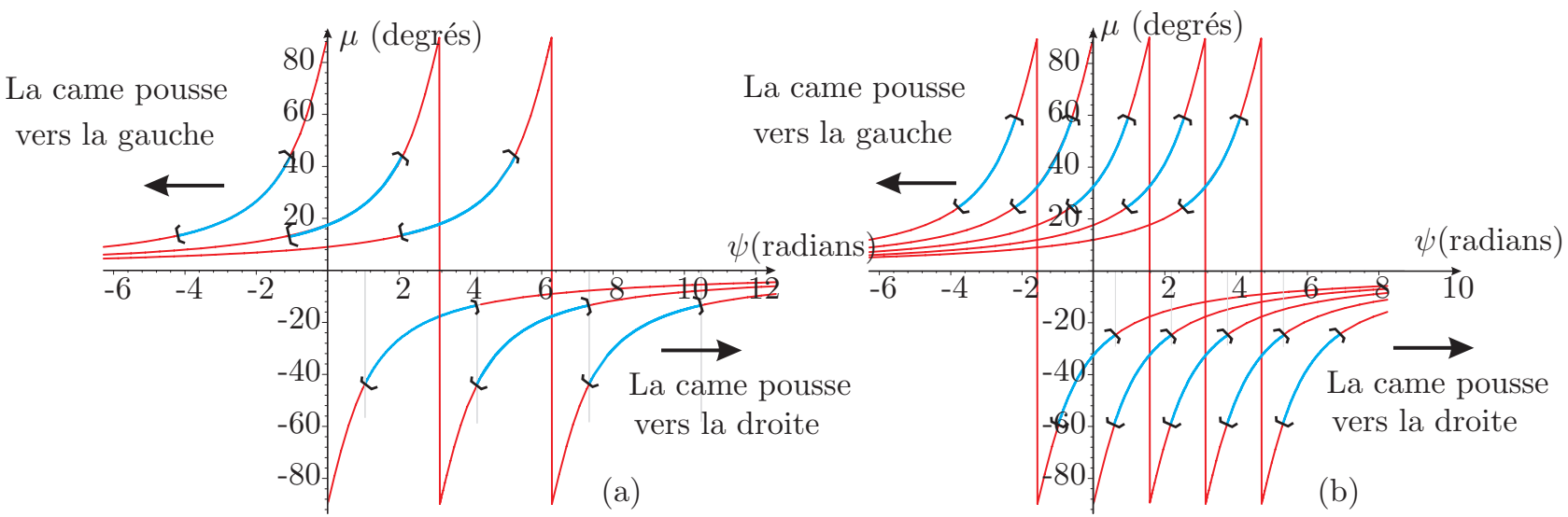

Fig. 11. Distribution de l'angle de pression pour deux cames conjuguées avec (a) un lobe et (b) deux lobes, avec $p=50 \mathrm{~mm}$, $a=10 \mathrm{~mm}$ et $e=9 \mathrm{~mm}$.

- Transmission à deux cames conjuguées : L'intervalle angulaire actif est :

$$
\frac{\pi}{n}-\Delta \leq \psi \leq \frac{2 \pi}{n}-\Delta
$$

En effet, la came conjuguée peut également mener le galet lorsque $0 \leq \psi \leq \pi / n-\Delta$; il existe donc un intervalle commun, pour $\pi / n \leq \psi \leq \pi / n-\Delta$, pendant lequel deux cames peuvent mener un galet. Dans cet intervalle, l'une des deux cames conjuguées peut mener un galet avec $|\mu|$ plus faible que pour la seconde. Dans ce cas, nous supposons que c'est la came avec $|\mu|$ le plus faible qui mène effectivement le galet. En conséquence, nous nous intéressons uniquement à la valeur de l'angle de pression dans l'intervalle $\pi / n-\Delta \leq \psi \leq 2 \pi / n-\Delta$, comme représenté dans la figure 11.

- Transmission à trois cames conjuguées : Pour la même raison, l'intervalle angulaire actif est :

$$
\frac{4 \pi}{3 n}-\Delta \leq \psi \leq \frac{2 \pi}{n}-\Delta
$$

Cet intervalle est $33 \%$ plus petit que celui obtenu avec une transmission à deux cames conjugées et la partie de l'intervalle supprimée est associée à l'angle de pression le plus élevé, comme représenté dans la figure 12.

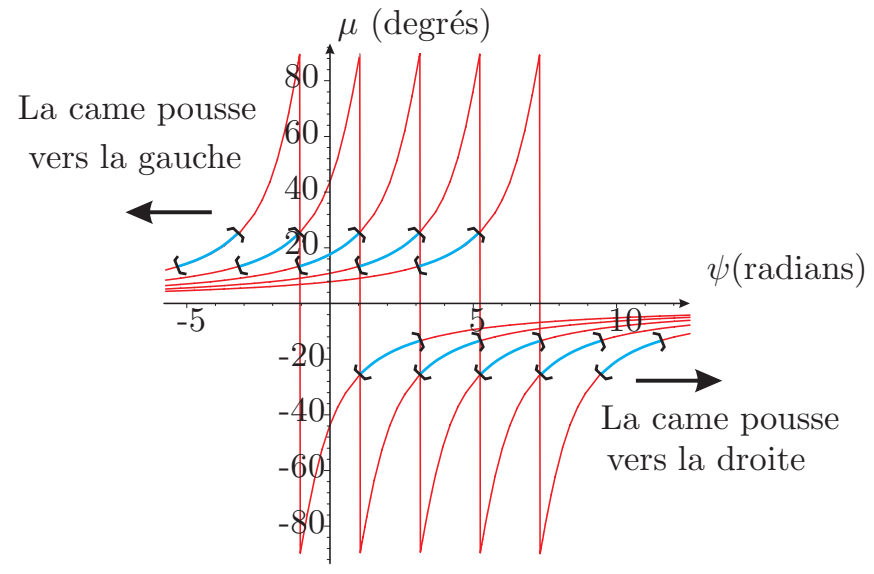

Fig. 12. Distribution de l'angle de pression pour trois cames conjuguées avec un lobe, $p=50 \mathrm{~mm}, a=10 \mathrm{~mm}$ et $e=9 \mathrm{~mm}$.

\subsection{Angle de pression et paramètres de conception}

Nous étudions ici l'influence des paramètres de conception $\eta$, $a$, et $n$ sur les valeurs de l'angle de pression, avec la came conduisant le galet, comme rapporté 


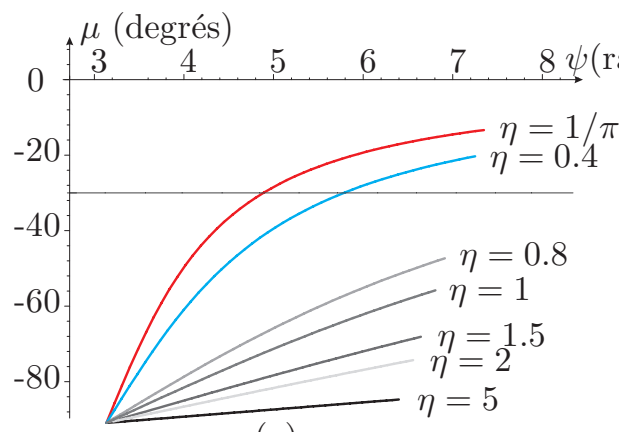

(a)

(c)

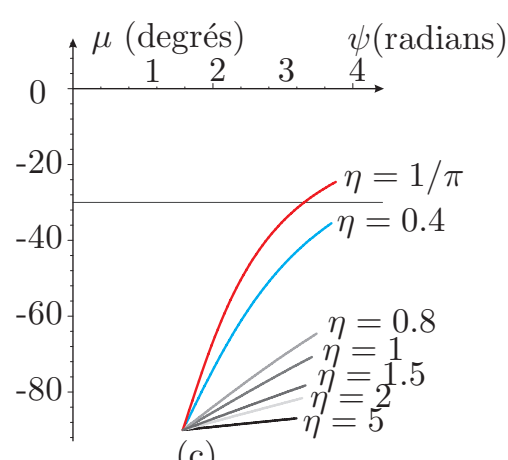

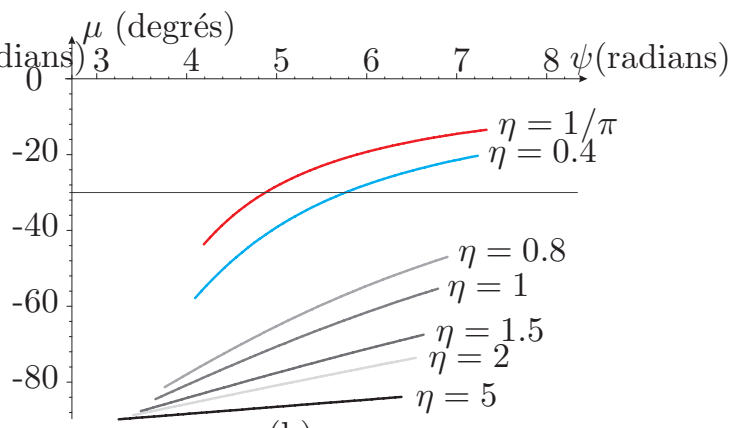

(b)

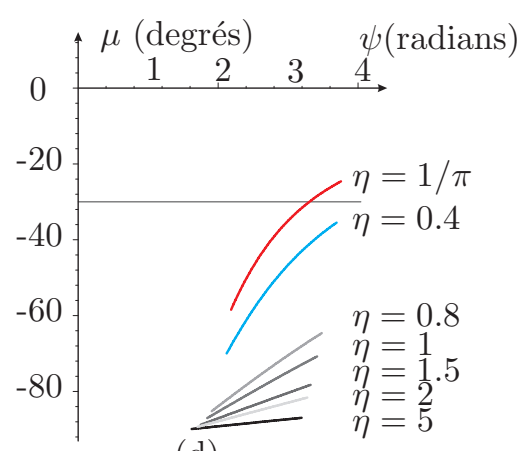

(d)

Fig. 13. Influence du paramètre $\eta$ sur l'angle de pression $\mu$ (en degrés), avec $p=50 \mathrm{~mm}$ et $a=10 \mathrm{~mm}$, pour (a) une came avec un seul lobe; (b) deux cames avec un seul lobe; (c) une came avec deux lobes; et (d) deux cames avec deux lobes.

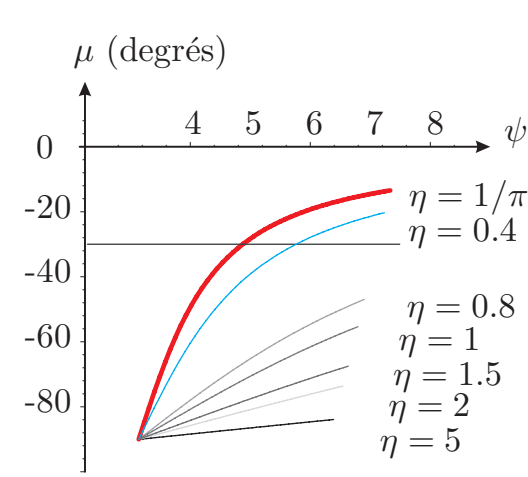

(a)

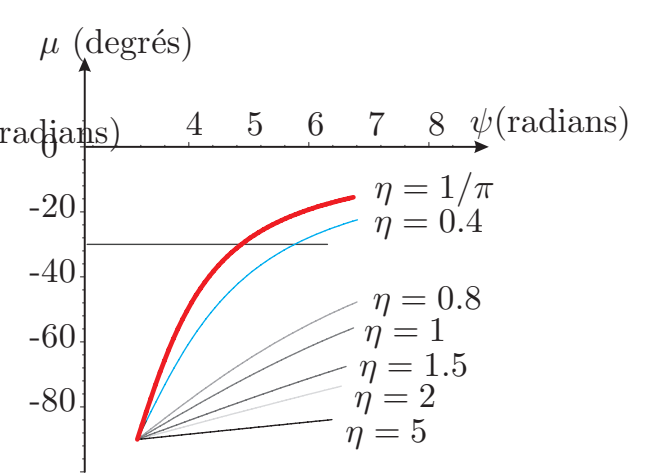

(b)

Fig. 14. Influence du paramètre $a$ sur l'angle de pression $\mu$ (en degrés), pour deux cames avec un seul lobe, avec $p=50$ mm et (a) $a=10 \mathrm{~mm}$ et (b) $a=25 \mathrm{~mm}$.

dans [13]. Nous étudions également l'influence du nombre de cames conjuguées sur la valeur de l'angle de pression.

\section{- Influence du paramètre $\eta$}

La figure 13 montre l'influence du paramètre $\eta$ sur l'angle de pression, avec les paramètres $a$ et $p$ fixes et pour plusieurs valeurs du nombre de cames et de lobes. À partir de ces figures, nous avons le résultat : Plus $\eta$ est petit, plus $|\mu|$ est faible, avec $\eta \geq 1 / \pi$, comme défini dans l'équation (15).

Ce résultat est identique si nous considérons une came simple avec plusieurs lobes ou une transmission avec deux ou trois cames conjuguées.

\section{- Influence du rayon de galet $a$}

Le paramètre $a$ n'apparaît pas dans l'expression de l'angle de pression, mais il influence la valeur de l'angle étendue $\Delta$, et par conséquent les limites de l'angle actif, comme montré dans la figure 14 pour deux valeurs de $a$. En calculant la valeur de l'angle étendue $\Delta$ pour plusieurs valeurs de $a$, nous notons que plus $a$ est grand, plus $|\Delta|$ est petit. En conséquence, puisque les frontières de l'angle de pression pour un système avec deux cames conjuguées sont $\pi / n-\Delta$ et $2 \pi / n-\Delta$ ou $4 \pi / 3 n-\Delta$ et $2 \pi / n-\Delta$ pour trois cames conjuguées, nous notons que lorsque nous augmentons $a,-\Delta$ diminue ou, d'une manière équivalente $\Delta$ augmente et la limite supérieure de l'intervalle angulaire 
actif diminue. Pourtant, c'est dans cette zone que $|\mu|$ est le plus faible. Nous avons ainsi le résultat : Plus a est petit, plus $|\mu|$ est faible.

Ce résultat est valide pour les transmissions avec une ou plusieurs cames et est indépendant du nombre de lobes.

\section{- Influence du nombre de lobes $n$}

En calculant la valeur de l'angle de pression pour plusieurs valeurs de $n$, comme représentées dans la figure 13, nous avons le résultat: Plus $n$ est petit, plus $|\mu|$ est faible. Avec deux lobes, le contact est perdu quand l'angle de pression est supérieur à $20^{\circ}$.

\section{- Influence du nombre de cames conjuguées $m$}

Les schémas 11(a) et 12 montrent que plus le nombre de cames est important, plus l'intervalle actif est faible. En particulier, l'augmentation du nombre de cames réduit la valeur maximale de $|\mu|$. Par conséquent, plus $m$ est grand, plus $|\mu|$ est faible.

\section{Conclusion et perspectives}

Deux stratégies de conception ont été étudiées dans cet article pour réduire l'angle de pression dans le mécanisme Slide-o-Cam. Les résultats obtenus se basent sur des contraintes technologiques et utilisent une formulation analytique. Pour la première fois, une étude complète sur le mécanisme Slide-o-Cam possédant plusieurs cames avec un ou plusieurs lobes a été réalisée. La solution optimale est trouvée $(i)$ pour des cames avec un seul lobe, $(i i)$ pour des mécanismes possédant le plus de cames conjuguées, (iii) pour la plus petite valeur du rayon $a$ des galets et $(i v)$ pour la plus petite valeur de $\eta$. Ces résultats peuvent maintenant servir de base pour une optimisation multi-critères et/ou multi-objectifs. Ainsi, pour réduire au minimum la déformation de l'axe des galets, le rayon $a$ doit être calculé pour résister aux efforts de flexion [8]. L'augmentation du nombre de lobes sur une came permet d'en diminuer sa taille, ce qui permet d'en réduire l'inertie mais au détriment d'une augmentation de l'angle de pression. Aussi, nous devons évaluer l'influence des variations des paramètres de conception sur l'angle de pression et étudier la conception de came possédant un profil concave sur des portions de came où elle ne travaille pas car cela permet l'obtention de valeurs de l'angle de pression encore plus faibles.

\section{Références}

[1] M.A. González-Palacios, J. Angeles, The design of a novel pure-rolling transmission to convert rotational into translational motion, J. Mechanical Design 125 (2003) 205-207

[2] D. Chablat, Ph. Wenger, Architecture Optimization of a 3-DOF Parallel Mechanism for Machining Applications, the orthoglide, IEEE Transactions on Robotics and Automation 19(3) (2003) 403-410

[3] J. Lampinen, Cam shape optimisation by genetic algorithm, Computer-Aided Design 35 (2003) 727-737

[4] Y. Zhang, J.-H., Shin, A Computational Approach to Profile Generation of Planar Cam Mechanisms, J. Mechanical Design 126-1 (2004) 183-188

[5] K.D., Bouzakis, S. Mitsi, J., Tsiafis, Computer-Aided Optimum Design and NC Milling of Planar Cam Mechanims, Int. J. Math. Tools Manufact. 37 (1997) 1131-1142

[6] J.E., Shigley, J.J., Uicker, 1980, Theory of Machines and Mechanisms, McGraw-Hill, New York, 1980

[7] S., Carra, R. Garziera, M., Pellegrini, Synthesis of cams with negative radius follower and evaluation of the pressure angles, Mechanism and Machine Theory 34 (2004) $1017-1032$

[8] J., Renotte D. Chablat, J., Angeles, The Design of a Novel Prismatic Drive for a Three-DOF ParallelKinematics Machine, ASME Design Engineering Technical Conferences, September-October 28-2, Salt Lake City, Utah, USA, 2004

[9] M.A. González-Palacios, J., Angeles, Cam Synthesis, Kluwer Academic Publishers B.V., Dordrecht, 1993

[10] K.J. Waldron, G.L., Kinzel, Kinematics, Dynamics, and Design of Machinery, John Wiley \& Sons, Inc., New York, 1999

[11] M.K., Lee, Design for Manufacturability of SpeedReduction Cam Mechanisms, M. Eng. Thesis, Dept. of Mechanical Engineering, McGill University, Montreal, 2001

[12] J. Angeles, C., López-Cajún, Optimization of Cam Mechanisms, Kluwer Academic Publishers B.V., Dordrecht, 1991

[13] W. Zhang, J. Angeles, A parametric study of planar cam-roller speed reducers, Transactions of the Canadian Society for Mechanical Engineering 28 (2004) 263-275 